\title{
O uso da eletroestimulação no nervo tibial posterior no tratamento da incontinência urinária
}

\section{Electrostimulation of the posterior tibial nerve in treatment of urinary incontinence \\ Uso de la electroestimulación en el nervio tibial posterior en el tratamiento de la incontinencia urinaria}

\author{
Andrelise Viana Rosa Tomasi'; Gesilani Júlia da Silva Honório ${ }^{I I}$; \\ Silvia Maria Azevedo dos Santos ${ }^{\text {III }}$; Karina Brongholi ${ }^{\text {IV }}$
}

\begin{abstract}
RESUMO: O objetivo deste estudo foi avaliar a eletroestimulação no nervo tibial posterior no tratamento da incontinência urinária de urgência ou mista. Trata-se de uma pesquisa quase experimental com oito pacientes, com diagnóstico de incontinência urinária de urgência ou mista, realizada no Estado de Santa Catarina, em 2010. A avaliação incluiu ficha de avaliação fisioterapêutica, diário miccional e questionário de qualidade de vida - King's Health Questionnaire. Foram realizados 12 atendimentos de 30 minutos com eletroestimulação no nervo tibial posterior. Os resultados mostram redução na frequência de perdas urinárias diurnas em $62 \%$ das pacientes, diminuição da frequência miccional noturna em $37,5 \%$ e redução da quantidade das perdas urinárias, de intensa para moderada e de moderada para leve. Não se observou diferença significativa nos escores do questionário de qualidade de vida após o tratamento. Conclui-se que o método estudado apresentou impacto positivo nas pacientes em relação às perdas involuntárias de urina e à quantidade de urina perdida.

Palavras-Chave: Incontinência urinária; eletroestimulação; nervo tibial; qualidade de vida.
\end{abstract}

\begin{abstract}
The aim of this study was to evaluate electrostimulation of the posterior tibial nerve in treating urgency or mixed urinary incontinence. This quasi-experimental study of eight patients with a diagnosis of urgency or mixed urinary incontinence, was conducted in Santa Catarina State, Brazil, in 2010. The assessment included a physical therapy evaluation form, bladder diary and King's Health Quality of Life Questionnaire. Twelve 30-minutes procedures of electro-stimulation of the posterior tibial nerve were performed. The results show reduced frequency of daytime urinary incontinence in $62 \%$ of the patients, decreased nocturnal urinary frequency in $37.5 \%$, and reduction of urinary leakage from severe to moderate and moderate to mild. No significant difference in quality of life questionnaire scores was observed after treatment. It was concluded that the method studied had positive impact on patients' involuntary urine loss and the amount of urine lost.
\end{abstract}

Keywords: Urinary incontinence; electrical stimulation; tibial nerve; quality of life.

RESUMEN: El objetivo de este estudio fue evaluar la electroestimulación del nervio tibial posterior en el tratamiento de la incontinencia urinaria de urgencia o mixta. Se trata de una investigación casi experimental con ocho pacientes, con diagnóstico de incontinencia urinaria de urgencia o mixta, realizada en el Estado de Santa Catarina - Brasil, en 2010. La evaluación incluye ficha de evaluación fisioterapéutica, diario miccional y cuestionario de calidad de vida King's Health Questionnaire. Se realizaron 12 atenciones de 30 minutos con electroestimulación del nervio tibial posterior. Los resultados muestran reducción en la frecuencia de pérdidas urinarias diurnas en $62 \%$ de las pacientes, disminución de la frecuencia miccional nocturna en $37,5 \%$ y reducción de la cantidad de orina de las pérdidas urinarias, de intensa a moderada y de moderada a leve. No se observó diferencia significativa en los resultados del cuestionario de calidad de vida después del tratamiento. Se concluye que el método estudiado presentó impacto positivo en las pacientes con relación a las pérdidas involuntarias de orina y a la cantidad de orina perdida.

Palabras Clave: Incontinencia urinaria; electroestimulación; nervio tibial; calidad de vida.

\section{INTRODUÇÃO}

A incontinência urinária (IU) é definida pela International Continence Society (ICS) e pela International Urogynecological Association (IUGA) como queixa de qualquer perda involuntária de urina ${ }^{1}$. A perda da continência urinária é uma circunstância desconfortável e embaraçosa, de origem multifatorial e geradora de exclusão, já que interfere na saúde física e mental das pessoas, comprometendo assim, sua qualidade de vida².

\footnotetext{
IFisioterapeuta. Mestranda do Programa de Pós-Graduação em Enfermagem da Universidade Federal de Santa Catarina. Participante do Grupo de Estudos sobre Cuidados em Saúde de Pessoas Idosas. Florianópolis, Santa Catarina, Brasil. E-mail: andrelisev@gmail.com.

IIFisioterapeuta. Doutoranda do Programa de Pós-Graduação em Enfermagem da Universidade Federal de Santa Catarina. Participante do Grupo de Estudos sobre Cuidados em Saúde de Pessoas Idosas. Docente do Curso de Fisioterapia do Centro Universitário Estácio de Sá de Santa Catarina. Florianópolis, Santa Catarina, Brasil. E-mail: gesilani@hotmail.com.

IIIEnfermeira. Doutora em Enfermagem. Docente do Departamento e Programa de Pós-Graduação em Enfermagem da Universidade Federal de Santa Catarina. Líder do Grupo de Estudos sobre Cuidados em Saúde de Pessoas Idosas. Florianópolis, Santa Catarina, Brasil. E-mail: silvia@ccs.ufsc.br. IVFisioterapeuta. Mestre em Neurociências. Docente do Curso de Fisioterapia do Centro Universitário Estácio de Sá de Santa Catarina. Florianópolis, Santa Catarina, Brasil. E-mail: karinab@live.estacio.br.
} 
A incontinência urinária de urgência (IUU) é definida como um desejo irresistível de urinar que dificilmente é suprimido ${ }^{3}$. Essa incontinência é causada por contrações involuntárias do detrusor (hiperatividade) acompanhadas pelo relaxamento uretral, durante a fase de enchimento vesical. A incontinência urinária mista (IUM) é caracterizada por sintomas da IUU associados à incontinência urinária de esforço (IUE), que por sua vez é descrita como a perda involuntária de urina na ausência de contração do detrusor, aos esforços, durante um aumento da pressão intra-abdomial ${ }^{4}$.

A frequência miccional aumentada e a noctúria geralmente acompanham o sintoma de urgência e são definidos respectivamente como, a queixa de apresentar repetidas miç̧ões durante o dia e acordar à noite uma ou mais vezes para urinar. Na literatura, é caracterizado o aumento da frequência como mais de oito micções durante o dia e noctúria como mais de duas micções durante a noite 5 .

Esses sintomas são comuns durante diagnóstico clínico. Outra modalidade que indica a IUU é o exame urodinâmico, em que há hiperatividade do detrusor durante a cistometria, podendo ser neurogênica ou idiopática. Admite-se que, em mais de $90 \%$ das vezes, a hiperatividade do detrusor é idiopática ${ }^{6}$. Outra forma de caracterizar a IU é o diário miccional. Esse diário pode ser considerado uma ferramenta importante para o correto diagnóstico da IU. Importantes variáveis podem ser extraídos do diário miccional: o débito urinário diurno e noturno, a capacidade vesical funcional, o número de micções durante o dia e a noite e o número de episódios de perda urinária?.

Como a IU tem grande impacto na qualidade de vida das pacientes, um instrumento para mensuração da qualidade de vida permitirá avaliar o resultado do tratamento escolhido e facilitará a comparação de estudos realizados em locais diferentes, utilizando tratamentos diferentes em populações semelhantes. Existem na literatura muitos questionários que abordam a qualidade de vida de mulheres com IU, sendo estes instrumentos genéricos ou específicos e que podem ser utilizados para tratamentos cirúrgicos ou conservadores. No Brasil, foi traduzido e validado o questionário King's Health Questionnaire (KHQ), que avalia tanto a presença de sintomas de IU, quanto seu impacto relativo. $\mathrm{O}$ uso de questionários como instrumentos de avaliação de qualidade de vida tem sido intensificado na pesquisa científica nos últimos anos. Com isso, valoriza-se a opinião dos pacientes sobre a sua condição de saúde ${ }^{8-10}$.

Há várias modalidades de tratamento para IU, desde os conservadores aos cirúrgicos. Uma das modalidades do tratamento conservador é a eletroestimulação, podendo ser aplicada em vários locais como pele perineal, estimulação esfincteriana, no dorso do pênis ou no nervo clitoridiano, métodos esses que foram testados em tipos diferentes de pacientes, tanto com instabilidade ou hiperreflexia do detrusor ${ }^{11}$.

O nervo tibial posterior é um nervo misto, contendo fibras motoras e sensoriais, saindo das raízes nervosas L4, L5, S1 a S3, compartilhando as mesmas raízes que inervam a bexiga. Consequentemente, a estimulação direta desse nervo deve inibir os aferentes S2-S3, suprimindo a atividade da bexiga ${ }^{12,13}$. Assim, uma nova forma de eletroestimulação pode vir ao encontro da melhora da hiperatividade do detrusor é a estimulação do nervo tibial posterior. Ainda não se conhece o efeito fisiológico da eletroestimulação transcutânea do nervo tibial posterior, mas acredita-se que tem a função de modular os estímulos que chegam a bexiga através da inervação recíproca. $\mathrm{O}$ impulso elétrico gerado pela corrente seria conduzido de forma retrógrada através do nervo tibial posterior até o plexo hipogástrico e, a partir deste até o detrusor, diminuindo suas contrações ${ }^{14}$.

Portanto, o objetivo deste estudo foi avaliar o uso da eletroestimulação no nervo tibial posterior, no tratamento da incontinência urinária de urgência ou mista.

\section{REVisão DE Literatura}

A eletroestimulação no nervo tibial posterior inibe a atividade da bexiga através da despolarização das fibras aferentes somáticas sacrais e lombares. A estimulação das fibras aferentes provê a inibição central dos motoneurônios pré-ganglionares vesicais diretamente na medula. A etiologia da hiperatividade vesical é desconhecida, mas a hipótese é que ocorre um desequilíbrio entre os impulsos inibitórios e excitatórios que comandam a bexiga, desta forma, a ação desta neuromodulação poderia restabelecer o equilíbrio no sistema nervoso central ${ }^{12,13}$.

A eletroestimulação do nervo tibial no tratamento para a IUU e IUM vem sendo estudada e discutida com eletrodos de inserção subcutâneos e superficiais, demonstrando resultados favoráveis, constituindo-se uma opção nos casos de contraindicação ou intolerância à eletroestimulação intracavitária, onde muitas mulheres apresentam desconforto com este tipo de procedimento. Portanto, a eletroestimulação no nervo tibial posterior com eletrodos adesivos não apresenta efeitos adversos, com facilidade de aplicação e os custos mínimos, podendo ser um importante método para tratamento em mulheres com IU ${ }^{15,16}$. Em alguns estudos que utilizaram a eletroestimulação com eletrodos subcutâneos, foram relatados eventos colaterais leves ou moderado como, incapacidade de tolerar a estimulação, cãibras nas pernas, resposta vasovagal e dormência durante a colocação da agulha ${ }^{17}$.

O tratamento conservador para a incontinência urinária de urgência ou mista com o uso da eletroestimulação no nervo tibial posterior utilizando eletrodos autoadesivos é uma técnica segura e pode reduzir o desconforto decorrente a perda de urina involuntária. 


\section{MÉTodos}

Trata-se de um estudo quase experimental. A população do estudo foi constituída por mulheres com histórico de queixa urinária, diagnosticadas através do exame urodinâmico, realizado por um médico urologista, durante os meses de julho de 2009 a janeiro de 2010, totalizando 30 pacientes. Destas, oito mulheres com incontinência urinária de urgência ou mista aceitaram participar do estudo.

Foram considerados critérios de exclusão: pósoperatório imediato de cirurgias uroginecológicas, uso de marcapasso, diabetes descompensada há mais de 6 meses e realização de outras formas de tratamento (utilização de medicamentos) para os sintomas urinários durante o estudo.

A coleta de dados foi realizada na Clínica Escola de Fisioterapia do Centro Universitário Estácio de Sá de Santa Catarina, no período de março a maio de 2010. Este estudo foi aprovado pelo Comitê de Ética e Pesquisa do Centro Universitário Estácio de Sá de Santa Catarina, parecer no 055/2009.

Os dados foram obtidos por meio de uma avaliação inicial com cada paciente, contendo dados de identificação, informações da incontinência urinária, como o tempo médio das queixas urinárias, os dados antropométricos (massa e altura), assim como histórico ginecológico e obstétrico. Após a avaliação inicial as pacientes foram orientadas a preencher corretamente $\mathrm{o}$ diário miccional, por três dias consecutivos. A coleta de dados do diário miccional incluiu as seguintes variáveis: frequência miccional diurna, noturna e perda urinária (frequência e quantidade). Também foi aplicado o questionário de qualidade de vida - KHQ.

Foram realizados 12 atendimentos, duas vezes por semana, com aplicação da eletroestimulação transcutânea no nervo tibial posterior, com duração de 30 minutos. Dois eletrodos autoadesivos foram colocados na região do tibial posterior, um próximo ao maléolo medial e o outro a 10 centímetros de distância, com a paciente em decúbito dorsal com os joelhos em extensão e o quadril em leve flexão e rotação externa. Uma corrente elétrica de 1 Hertz $(\mathrm{Hz})$ e 200 milissegundos foi programada no aparelho até que se observou uma flexão dos dedos. Uma vez localizado o nervo tibial posterior, a frequência do aparelho foi alterada para $20 \mathrm{~Hz}$ e a intensidade de acordo com a tolerância da paciente foi de 0-10 miliampere (mA), mas abaixo do limiar motor do nervo ${ }^{18}$.

Todos os atendimentos foram realizados na Clínica Escola de Fisioterapia do Centro Universitário Estácio de Sá de Santa Catarina. Ao final do tratamento, as pacientes foram reavaliadas com os mesmos instrumentos utilizados na avaliação inicial.

A identificação das pacientes foi mantida em anonimato de acordo com as diretrizes e normas de pesquisa envolvendo seres humanos estabelecidos pela
Resolução no 196 do Conselho Nacional de Saúde de 10 de outubro de 1996. As pacientes assinaram um Termo de Consentimento Livre e Esclarecido, após esclarecimentos dos objetivos e procedimentos que seriam realizados.

Para a descrição dos resultados do estudo foram considerados os seguintes parâmetros: na análise do diário miccional foram observadas mudanças dos parâmetros miccionais. Estas mudanças serviram como critério de melhora do quadro de incontinência, como a diminuição da frequência miccional diurna, noturna e perda de urina.

Para a análise estatística da diferença da frequência miccional diurna, noturna, perdas urinárias pré e pós-tratamento, e escores dos domínios do questionário de qualidade de vida, realizou-se o teste de Wilcoxon, com nível de significância de p<0,05.

\section{Resultados e Discussão}

A média de idade das mulheres estudadas foi de 62,4 anos. Esses resultados estão de acordo com a literatura, a média de idade nos estudos estão entre 56, 4 ou 61,5 anos. Portanto, a maior frequência em mulheres acima de $50 \operatorname{anos}^{19,20}$. Isso também se confirma no presente estudo, pois somente uma paciente tinha menos de 50 anos, e todas as outras estavam acima desta idade.

O estilo de vida e intervenções comportamentais no tratamento da IU estão associados com maior risco para o aparecimento dos sintomas, sendo a obesidade um fator de risco em homens e mulheres, onde o aumento da pressão intra-abdominal pode levar a um estresse crônico sobre o assoalho pélvico ${ }^{21}$. Isto confirma os resultados obtidos através das participantes desta pesquisa, em que a média do índice de massa corporal foi de $27,8 \mathrm{~kg} / \mathrm{m}^{2}$, com quatro pacientes apresentando sobrepeso e duas pacientes com obesidade grau I.

Em relação ao tempo de duração das queixas urinárias nas mulheres participantes em outro estudo, a média é de no máximo 4,5 anos ${ }^{12}$. Desta forma, observa-se que as participantes deste estudo ficaram expostas há um tempo maior com média de 5,1 anos. O motivo da demora em procurar soluções para os diversos tipos de IU ocorre em função da vergonha e do constrangimento sentido por parte dos pacientes, pelo desconhecimento ou habilidade por parte dos profissionais da saúde, sendo que durante as consultas médicas rotineiras esse assunto não costuma ser falado ou investigado 22 .

Neste estudo, a maior parte das pacientes estavam no climatério, corroborando com outros estudos divulgados na literatura, indicando que a IU se apresenta em 35\% das mulheres no climatério ${ }^{23,24}$. Outro fator de risco são os procedimentos cirúrgicos ginecológicos, principalmente a histerectomia, as mulheres 
são submetidas com maior frequência, pois a retirada do útero compromete o bom funcionamento da musculatura do assoalho pélvico, podendo causar danos na sustentação da bexiga e da uretra, e ser responsável pelo aumento da prevalência da IU ${ }^{25}$. Neste estudo, foi identificado que 07 mulheres realizaram algum tipo de cirurgia ginecológica ou urológica, sendo que destas, 06 realizaram histerectomia. Com base no histórico obstétrico, constatou-se que somente uma paciente não teve nenhuma gestação, as outras eram multigestas. Em relação ao parto, as participantes realizaram um ou mais partos vaginais. $\mathrm{O}$ parto pode levar a algumas lesões perineais consecutivas à distensão do períneo, podendo afetar o elemento cutâneo (pele, mucosa vaginal), os elementos fibrosos ligamentares, o elemento nervoso e as estruturas musculares. Muitos fatores, como a multiparidade, fetos macrossômicos, uma fase prolongada do trabalho de parto e o uso de instrumento para assistência como fórceps, têm sido associados como fatores de risco aumentado para $\mathrm{IU}^{26}$.

Portanto, quando comparado com o parto cesáreo, o parto vaginal isoladamente não é o causador da IU e sim, quando associado às lesões ou traumas do assoalho pélvico e a outros fatores de risco ${ }^{27}$. No que se refere ao desfecho perineal, em um estudo observou-se que em 6.365 partos vaginais, destes 4.542 tiveram algum tipo de laceração espontânea ou episiotomia ${ }^{28}$. Esse estudo demonstra a grande prevalência de lesões no períneo durante o parto vaginal, considerado fator de risco para os sintomas da IU. Esse tipo de lesão cada vez mais tem que ser pesquisado, pois em um estudo foi observado que, 6 meses após o parto vaginal, as mulheres relataram ainda dor na região do períneo e alterações na cicatrização da episiotomia ${ }^{29}$. Neste estudo identificou-se redução das frequências diurnas e noturnas após os atendimentos, sem diferença significativa. Em relação a perdas urinárias entre as participantes, estas ocorriam no máximo sete vezes antes do tratamento, e ao final, houve redução para duas perdas. Das participantes, três não relataram perda ao final do tratamento e as outras cinco destacaram diminuição da quantidade da perda. Estes dados foram coletados através do diário miccional, preenchido pelas pacientes antes e após o tratamento, conforme mostra a Tabela 1.

TABELA 1: Frequência urinária diurna, noturna e as perdas urinárias obtidas através do diário miccional. Santa Catarina, Brasil, 2010.

\begin{tabular}{lccc}
\hline \multicolumn{1}{c}{ Variáveis } & $\begin{array}{c}\text { Antes } \\
\text { (mediana) }\end{array}$ & $\begin{array}{c}\text { Após } \\
\text { (mediana) }\end{array}$ & $\mathrm{p}^{(*)}$ \\
\hline Frequência diurna & 7 & 6 & 0,071 \\
Frequência noturna & 2 & 1 & 0,1 \\
Perda urinária & 3 & 2 & 0,432 \\
\hline
\end{tabular}

Teste de Wilcoxon, $\mathrm{p}^{(*)} \leq 0,05$
Sobre os dados relacionados à perda urinária no presente estudo, houve diminuição da frequência miccional diurna em 5 (62\%) participantes. Este resultado está de acordo com a literatura, pois se encontra, entre os índices de melhora, 50\% a 70\% dos participantes, registrados no diário miccional ${ }^{30}$.

Em relação à noctúria, é difícil comparar com outros resultados da literatura, pois há diferenças entre as características das amostras. Em estudo relacionado com problemas de origem neurogênica, houve queda de mais de $50 \%$ na frequência urinária, durante a noite, em 20 pacientes estudados, ao contrário do presente estudo ${ }^{12}$. Já em outro estudo foi observado, uma redução da noctúria, durante o tratamento, de 2,38 micções por noite para 1,14 micções, sendo este resultado significante ${ }^{31}$.

Sobre a eletroestimulação para a IU, observa-se que sua utilização no nervo tibial posterior produziu uma diminuição significativa do quadro em alguns estudos descritos na literatura. Estes achados se encontram em artigos que não utilizaram os mesmos critérios de inclusão do presente estudo, mas que realizaram o tratamento com pacientes com IU de origem idiopática ${ }^{11,12,32}$.

No presente estudo, 5 (62\%) participantes apresentaram perda de urina, após a aplicação da eletroestimulação no nervo tibial posterior, mas foi observada uma melhora da quantidade, de intensa para moderada e de moderada para leve. Nesta pesquisa, foram utilizados eletrodos autoadesivos, o que difere em relação a outros estudos que realizaram o procedimento com eletrodos de agulha.

Em estudo descrito na literatura, com abordagem de 33 participantes, com 12 semanas de tratamento e após uma intervenção aos 6 e 12 meses de tratamento, foi verificada uma melhora sustentada de 12 semanas a 6 meses para $94 \%$ dos participantes, e de 12 meses para 96\% dos indivíduos avaliados, não ocorrendo nenhum evento adverso grave, após o período de um ano de tratamento ${ }^{33}$.

Em relação ao questionário de qualidade de vida aplicado durante o estudo, não se observou diferença estatística significativa em nenhum dos escores dos nove domínios, antes e após o tratamento, conforme mostra a Tabela 2.

O domínio de limitações sociais não teve alteração após o tratamento, e nos outros domínios como, relações pessoais, emoções, sono, disposição e medidas de gravidade, houve melhora não significativa após o tratamento. Vale destacar um estudo no qual foi obervado que à medida que se favorece a manutenção do convívio social, melhora a qualidade de vida das pessoas, o que não foi observado no presente estudo ${ }^{34}$.

Em pesquisa realizada com o mesmo questionário do presente estudo, em relação aos escores do questionário $\mathrm{KHQ}$, observou-se que somente o domínio percepção geral da saúde não demonstrou variação 
TABELA 2: Comparação dos escores da qualidade de vida, utilizando o questionário King's Health Questionnaire (KHQ), antes e após o tratamento. Santa Catarina, Brasil, 2010.

\begin{tabular}{lccc}
\hline \multicolumn{1}{c}{ Domínios do KHG } & $\begin{array}{c}\text { Antes } \\
\text { (média } \pm \text { DP) }\end{array}$ & $\begin{array}{c}\text { Após } \\
\text { (média } \pm \text { DP) }\end{array}$ & $\mathbf{p}^{(*)}$ \\
\hline Percepção geral da saúde & $31,3 \pm 17,7$ & $42,9 \pm 18,9$ & 0,211 \\
Impacto da incontinência & $54,2 \pm 35,4$ & $50,0 \pm 30,9$ & 0,394 \\
Limitações das atividades diárias & $33,3 \pm 32,1$ & $29,2 \pm 44,3$ & 0,393 \\
Limitações físicas & $33,3 \pm 34,5$ & $31,3 \pm 40,3$ & 0,25 \\
Limitações sociais & $18,1 \pm 26,5$ & $18,1 \pm 28,4$ & 0,5 \\
Relações pessoais & $83,3 \pm 0$ & $50,0 \pm 23,6$ & - \\
Emoções & $38,9 \pm 32,0$ & $26,4 \pm 33,0$ & 0,172 \\
Sono e disposição & $39,6 \pm 35,6$ & $29,2 \pm 34,2$ & 0,337 \\
Medidas de gravidade & $46,9 \pm 22,7$ & $39,6 \pm 25,9$ & 0,376 \\
\hline
\end{tabular}

$K H Q-K i n g ' s$ Health Questionnaire; $p\left(^{*}\right) \leq 0,05$

significativa após a aplicação da eletroestimulação. Já nos outros domínios, observou-se uma melhora significativa no valor dos escores ${ }^{35}$. Em contrapartida, um outro estudo apresentou melhora nos domínios de limitação física e sono, e nos outros domínios do questionário KHQ não foram observados alterações após o tratamento ${ }^{36}$.

O impacto da IU sobre a vida de homens e mulheres inclui efeitos prejudiciais para atividades sociais, profissionais, de lazer, saúde sexual e relações com os membros da família. Os resultados obtidos em outros estudos indicam que o impacto da IU é maior para a saúde física do que para a saúde mental ${ }^{21,37}$. Apesar da IU ser uma queixa frequente nas mulheres, muitas acabam não relatando aos seus médicos, dificultando a procura por serviços especializados. Dessa forma, adesão a um tratamento conservador acaba sendo baixo, o que limita as pesquisas. E, também, o fato de o tratamento conservador requerer um tempo maior para apresentar os primeiros resultados e perceber de forma mais efetiva o controle da incontinência ${ }^{22}$.

\section{ConClusão}

O tratamento com eletroestimulação no nervo tibial posterior apresentou um resultado positivo em relação à frequência miccional diurna e noturna, e, também, em relação às perdas involuntárias das participantes do estudo. Com relação ao questionário de qualidade de vida, obtiveram-se alterações positivas, porém não estatisticamente significantes.

Este tipo de tratamento proposto para pacientes incontinentes é viável, como tratamento conservador da incontinência urinária, devido ao seu baixo custo e à ausência dos efeitos colaterais.

Deve-se ressaltar, no entanto, que esses resultados foram obtidos com um universo amostral relativamente pequeno, sendo necessário novos estudos sobre o uso da eletroestimulação no nervo tibial posterior, bem como verificar o tempo de permanência de tais achados.

\section{REFERÊNCIAS}

1.Haylen BT, Ridder D, Freeman RM, Swift SE, Berghmans B, Lee J, et al. An International Urogynecological Association (IUGA) / International Continence Society (ICS) joint report on the terminology for female pelvic floor dysfunction. Int Urogynecol J. 2010; 21: 5-26.

2.Felderner Jr PC, Sartori MGFS, Lima GR, Baracat EC, Girão MJBC. Diagnóstico clínico e subsidiário da incontinência urinária. Rev Bras Ginecol Obstet. 2006; 28:54-62. 3. Fujihara A, Ukimura $\mathrm{O}$, Hongo $\mathrm{H}$, Iwata T, Veda T, Matsugasumi T, et al. Urge perception indexo of bladder hypersensitivity. J Urol. 2013; 189:1797-803.

4.Dwyer PL. Differentiating stress urinary incontinence from urge urinary incontinence. Int J Gynecol Obstet. 2004; 86:17-24.

5.Klingler HC, Schmidbauer J, Maeberger M. Use of peripheral neuromodulation of S3 region for treatment of detrusor overactivity: a urodynamic - based study. Urology. 2000; 56:766-71.

6.Arruda RM, Souza GO, Castro RA, Sartoria MGF, Baracat EC, Girões MJBC. Hiperatividade do detrusor: comparação entre oxibutinina, eletroestimulação funcional do assoalho pélvico e exercícios perineais. Estudo randomizado. Rev Bras Ginecol Obstet. 2007; 29:452-8. 7.Brown JS, McNaughton KS, Wyman JF, Burgio KL, Harkaway R, Bergner D, et al. Measurement characteristics of a voiding diary for use by men and women with overactive bladder. Urology. 2003; 61:802-9.

8.Fonseca ESM, Camargo ALM, Castro RA, Sartori MGFS, Fonseca MCM, Lima GR, et al. Validação do questionário de qualidade de vida (King's Health Questionnaire) em mulheres brasileiras com incontinência urinária. Rev Bras Ginecol Obstet. 2005; 27:235-42.

9.Rett MT, Simões JA, Herrmann V, Gurgel MSC, Moraes SS. Qualidade de vida em mulheres após tratamento da incontinência urinária de esforço com fisioterapia. Rev Bras Ginecol Obstet. 2007; 29:134-40.

10.Tamanini JTN, D'Ancona CAL, Botega NJ, Rodrigues Netto Jr N. Validação do King's Health Questionnaire para o português em mulheres com incontinência urinária. Rev Saude Publica. 2003; 37:203-11. 
11.Amarenco G, Ismael SS, Even-Schneider A, Raibaut P, Demaille-Wlodyka S, Parratte B, et al. Urodynamic effect of acute transcutaneous posterior tibial nerve stimulation in overactive bladder. J Urol. 2003; 169:2210-5. 12.Vandoninck V, Van Balken MR, Agro EF, Petta F, Caltagirone C, Heesakkers JPFA, et al. Posterior tibial nerve stimulation in the treatment of urge incontinence. Neurourol Urodyn. 2003; 22:17-23.

13. Vandoninck V, Van Balken MR, Agro EF, Heesakkers JPFA, Debruyne FMJ, Kiemeney LALM, et al. Posterior tibial nerve stimulation in the treatment of voiding dysfunction: urodynamic data. Neurourol Urodyn. 2004; 23:246-51. 14. Van Balken MR, Vergunst H, Bemelmans BLH. The use electrical devices for the treatment of bladder dysfunction: a review of methods. J Urol. 2004; 172:846-51. 15.Franco MM, Souza FO, Vasconcelos ECLM, Freitas MMS, Ferreira CHJ. Avaliação da qualidade de vida e da perda urinária de mulheres com bexiga hiperativa tratadas com eletroestimulação transvaginal ou do nervo tibial. Fisioter Pesq. 2011; 18:145-50.

16.Booth J, Hagen S, McClurg D, Norton C, MacInnes C, Collins B, et al. A feasibility study of transcutaneous posterior tibial nerve stimulation for bladder and bowel dysfunction in elderly adults in residential care. Am J Med Assoc Dir. 2013; 14:270-4.

17.Moossdorff-Steinhauser HFA, Berghmans B. Effects of percutaneous tibial nerve stimulation on adult patients with overactive bladder syndrome: a systematic review. Neurourol Urodyn. 2013; 32:206-14.

18.Van Der Pal F, Van Balken MR, Heesakkers JPFA, Debruyne FMJ, Kiemeney LALM, Bemelmans BLH. Correlation between quality of life and voiding variables in patients treated with percutaneous tibial nerve stimulation. BJU International. 2006; 97:113-6.

19.Brown JS, Bradley CS, Subak LL, Richter HE, Kraus SR, Brubaker L, et al. The sensitivity and specificity of a simple test to distinguish between urge and stress urinary incontinence. Ann Intern Med. 2006; 144:715-23.

20.Marchal C, Herrera B, Antuña F, Saez F, Perez J, Castilho E, et al. Percutaneous tibial nerve stimulation in treatment of overactive bladder: when should retreatment be started? Urology. 2011; 78:1046-50.

21. Wyman JF, Burgio KL, Newman DK. Practical aspects of lifestyle modifications and behavioural interventions in the treatment of overactive bladder and urgency urinary incontinence. Int J Clin Pract. 2009; 63:1177-91.

22.Honório MO, Santos SMA. A rede de apoio ao paciente incontinente: a busca por suporte e tratamentos. Rev enferm UERJ. 2010; 18:383-8.

23.Matheus LM, Mazzari CF, Mesquita RA, Oliveira J. Influência dos exercícios perineais e dos cones vaginais, associados à correção postural, no tratamento da incontinência urinária feminina. Rev Bras Fisioter. 2006; 10:387-92.
24.Guarisi T, Net AMP, Osis MJ, Pedro AO, Paiva LHC, Faúndes A. Incontinência urinária entre mulheres climatéricas brasileiras: inquérito domiciliar. Rev Saude Publica. 2001; 35:428-35.

25.Moller LA, Lose G, Jorgensen T. Risk factors for lower urinary tract symptoms in women 40 to 60 years of age. Obstet Gynecol. 2000; 96:446-51.

26.Higa R, Lopes MHBM, Reis MJ. Fatores de risco para a incontinência urinária na mulher. Rev Esc Enferm USP. 2008; 42:187-92.

27.Fenner DE, Genberg B, Brahma P, Marek L, Delancey JOL. Fecal and urinary incontinence after vaginal delivery with anal sphincter disruption in an obstetrics unit in the United States. Am J Obstet Gynecol. 2003; 189:1543-50. 28. Riesco MLG, Costa ASC, Almeida SFS, Basile ALO, Oliveira SMJV. Episiotomia, laceração e integridade perineal em partos normais: análise de fatores associados. Rev enferm UERJ. 2011; 19:77-83.

29.Silva, NLS, Oliveira SMJV, Silva FMB, Santos JO. Dispareunia, dor perineal e cicatrização após episiotomia. Rev enferm UERJ. 2013; 21:216-20.

30.Van Balken MR, Vergunst H, Bemelmans BLH. Prognostic factors for successful percutaneous tibial nerve stimulation. Eur Urol. 2006; 49:360-5.

31.Bellette PO, Rodrigues-Palma PC, Hermann V, Riccetto C, Bigozzi M, Olivares JM. Posterior tibial nerve stimulation in the management of overactive bladder: a prospective and controlled study. Actas Urol Esp. 2009; 33:58-63.

32.De Gennaro M, Capitanucci ML, Mastracci P, Silveri M, Gatti C, Mosiello G. Percutaneous tibial nerve neuromodulation is well tolerated in children and effective for treating refractory vesical dysfunction. J Urol. 2004; 171:1911-3.

33.MacDiarmid SA, Peters KM, Shobeiri SA, Wooldridge LS, Rovner ES, Leong FC, et al. Long-term durability of percutaneous tibial nerve stimulation for the treatment of overactive bladder. J Urol. 2010; 183:234-40.

34.Moliterno ACM, Faller JW, Borghi AC, Marcon SS, Carreira L. Viver em família e qualidade de vida de idosos da universidade aberta da terceira idade. Rev enferm UERJ. 2012; 20:179-84.

35. Fischer-Sgrott FO, Manffra EF, Busato Junior WFS. Qualidade de vida de mulheres com bexiga hiperativa refratária tratadas com estimulação elétrica do nervo tibial posterior. Rev Bras Fisioter. 2009; 13:480-6.

36. Onal M, Ugurlucan FG, Yalcin O. The effects of posterior tibial nerve stimulation on refractory overactive bladder syndrome and bladder circulation. Arch Gynecol Obstet. 2012; 286:1453-7.

37.Paick JS, Kim SW, Oh SJ, Ku JH. A generic healthrelated quality of life instrument, the medical outcomes study short form-36, in women with urinary incontinence. Eur J Obstet Gynecol Reprod Biol. 2007; 130:18-24. 